\section{Passive and active seismic isolation for gravitational radiation detectors and other instruments}

\author{
N A Robertson + , R W P Dreverł, I Kerr and J Hough \\ Department of Natural Philosophy, University of Glasgow, \\ Glasgow G12 8QQ, UK
}

\section{Received 26 February 1982, in final form 20 May 1982}

\begin{abstract}
Some new passive and active methods for reducing the effects of seismic disturbances on suspended masses are described, with special reference to gravitational radiation detectors in which differential horizontal motions of two or more suspended test masses are monitored. In these methods it is important to be able to determine horizontal seismic accelerations independent of tilts of the ground. Measurement of changes in inclination of the suspension wire of a test mass, relative to a direction defined by a reference arm of long period of oscillation, makes it possible to carry this out over the frequency range of interest for earth-based gravitational radiation detectors. The signal obtained can then be used to compensate for the effects of seismic disturbances on the test mass if necessary. Alternatively the signal corresponding to horizontal acceleration can be used to move the point from which the test mass is suspended in such a way as to reduce the effect of the seismic disturbance and also damp pendulum motions of the suspended test mass. Experimental work with an active anti-seismic system of this type is described.
\end{abstract}

\section{Introduction}

At Glasgow University a separated mass gravitational wave detector incorporating optical displacement sensing is being developed (Drever et al 1980) and some new methods for reducing the effects of seismic disturbances on the detector are being investigated. Gravitational radiation is predicted to induce tidal strains in space, the most significant sources for detection being astrophysical in origin. Signals may be searched for by monitoring changes $\Delta L$ in the separation $L$ of essentially free test masses. In practice if two baselines are formed perpendicular to each other between three masses suspended as pendulums, a gravitational wave pulse of suitable polarisation and direction of incidence should cause a fluctuation in the relative length of the two baselines, this length being monitored by multiple beam or resonant cavity laser interferometry (Drever et al 1981). Currently at Glasgow a baseline of $10 \mathrm{~m}$ is being used but this may be significantly extended in the future. Possible advantages of separated mass detectors over resonant bar detectors include their potentially large bandwidth, and this should allow gravitational wave signals to be searched for over a wide range of frequencies from tens of $\mathrm{Hz}$ to several $\mathrm{kHz}$ where pulsed strains with spectral density of the order of $10^{-20}(\mathrm{~Hz})^{-1 / 2}$ to $10^{-22}(\mathrm{~Hz})^{-1 / 2}$ might be expected at a rate of approximately one event per month (Thorne 1980). However

$\dagger$ Now at Blackett Laboratory, Imperial College of Science and Technology, London, UK.

\$Also at California Institute of Technology, Pasadena, California, USA. background effects such as those due to ground-borne noise must then be kept at a suitably low level.

For the isolation of gravitational wave detectors against ground-borne noise two factors should be noted. Firstly, if the test masses in the detector are hung as pendulums, ground noise acts on the masses through its effect on their points of suspension while gravitational wave signals act directly on the masses themselves as well as on their points of suspension. Thus the effects of gravitational radiation and ground movements can be separated from each other, and the latter can be reduced, for example by isolating the suspension points of the pendulums from the ground, without affecting the sensitivity of the apparatus to gravitational waves. Secondly, for gravitational radiation detectors operating in a horizontal plane, seismically induced horizontal motions of the test masses are only caused, at least to first order, by true horizontal movements of the ground, and it is important that any technique for seismic isolation should reduce the effect of horizontal seismic movements without introducing any coupling to other ground movements. In this paper a relatively simple method of sensing true horizontal seismic disturbances independent of tilt of the ground over the frequency range of interest is introduced, and possible isolation techniques based on this are discussed.

\section{The reduction of seismic noise in gravitational wave detectors} In most of the early gravitational wave detectors, simple passive isolation techniques were sufficient to reduce the effect of seismic noise below that of other noise sources (see e.g. Weber 1969). These techniques included suspending the resonant bar which forms the antenna of the gravitational wave detector by a wire and isolating the point of suspension from the ground by stacks of alternating layers of lead and rubber which act as vibration filters. Air springs have also been used to give additional high-frequency isolation.

These techniques are adequate for operating frequencies around $1 \mathrm{kHz}$ but separated mass detectors are potentially capable of experiments down to much lower frequencies and may demand a much greater degree of isolation. There are a number of reasons for this. The spectrum of seismic noise is such that ground motions from both natural and man-made sources are greater at low frequencies. Also a single stage of isolation by a simple system such as a pendulum suspension or a mass and spring isolator of resonant angular frequency $\omega_{0}$ attenuates ground displacements occurring at angular frequency $\omega \gg \omega_{0}$ by a factor $\left(\omega / \omega_{0}\right)^{2}$ when damping is negligible. Thus the isolation provided decreases with decreasing frequency. Further, the test masses in a long-baseline detector have to be suspended independently and so the intrinsic common mode rejection of a resonant bar detector suspended from a single point is lost. At a quiet laboratory site ground horizontal motions are expected to be of the order of $10^{-11} \mathrm{~m}(\mathrm{~Hz})^{-1 / 2}$ at $25 \mathrm{~Hz}$ falling to $5 \times$ $10^{-15} \mathrm{~m}(\mathrm{~Hz})^{-1 / 2}$ at $1 \mathrm{kHz}$ (see for example Weiss 1972). For a mass hung on a pendulum suspension of high quality factor and natural resonant frequency of $1 \mathrm{~Hz}$ the resulting motion will vary from $10^{-14} \mathrm{~m}(\mathrm{~Hz})^{-1 / 2}$ at $25 \mathrm{~Hz}$ to $5 \times 10^{-21} \mathrm{~m}(\mathrm{~Hz})^{-1 / 2}$ at $1 \mathrm{kHz}$ and at the low-frequency end this is four orders of magnitude larger than the predicted photon noise limited sensitivity (approx. $5 \times 10^{-19} \mathrm{~m}(\mathrm{~Hz})^{-1 / 2}$ ) of interferometric separated mass detectors.

Other techniques for reducing seismic noise signals can also be implemented. One possible approach could be to suspend each test mass from a servo-controlled inertial platform such as that described by Lorenzini (1972) which uses accelerometers and other sensors to monitor the six degrees of freedom which may be important. Another method would be to monitor interferometrically the relative horizontal movements of the separated points of suspension of the test masses, deduce their 
effects on the relative separations of the test masses and subtract the resulting signal from the main interferometer output. However, a simpler approach may be to obtain a measure of the motion of each test mass separately due to ground motions and subtract these signals from the main interferometer output. In principle the horizontal motion signals might be obtained by means of accelerometers mounted on the test masses but such a system is limited by the sensitivity of the relatively small accelerometers which could be used and the need to separate signals due to rocking motions of the masses from true horizontal signals.

The required signals could be obtained from accelerometers mounted at the points of suspension of the masses, provided the effects of tilts of the ground could be removed from the accelerometer output signals. This could be achieved with gyroscopes or combinations of accelerometers as are used in servo-controlled inertial platforms. However, we propose here a much simpler and more practical arrangement in which measurements of changes of inclination of the wires suspending the test masses are made relative to freely suspended reference arms which are arranged to have a natural period of oscillation much longer than the timescales of interest in the experiment. From these measurements, assuming that the masses of the suspension wires are negligible, the horizontal components of acceleration of the masses can be deduced over the relevant timescale of approximately $10^{-1} \mathrm{~s}$ to $10^{-3} \mathrm{~s}$ independent of the effects of ground tilting movements.

\section{Use of a reference arm}

A body suspended freely and precisely at its centre of gravity would retain its orientation unaffected by seismic vibrations, and could provide a perfect reference for the determination of horizontal seismic movements independent of tilts of the ground. By measuring the inclination of the suspension wire relative to this, as indicated by the difference in distance $\left(x_{a}-x_{b}\right)$ in figure 1 , one horizontal component of seismic noise could in principle be completely removed from the observed data. In practice, if the suspension point of the reference arm does not perfectly coincide with its centre of gravity, so that the arm has a resonant angular frequency $\omega_{\mathrm{r}}$, then seismic disturbances may cause it to move slightly. It can be shown that at angular frequencies much greater than $\omega_{\mathrm{r}}$, and provided damping is negligible, the residual effect of horizontal disturbances on the main interferometer output signal, after the necessary subtractions have been carried out, is as if each test mass has been isolated by a pendulum suspension of resonant angular frequency $\omega_{r}$. Damping of the

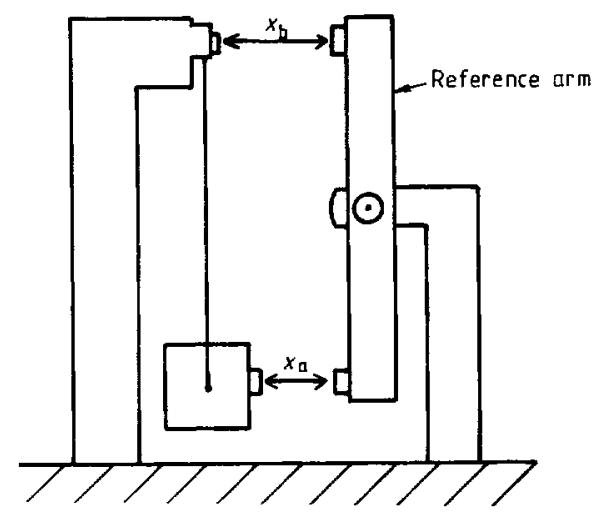

Figure 1. A possible arrangement for monitoring horizontal accelerations of a test mass induced by seismic motion. reference arm to the ground does not significantly degrade isolation against horizontal disturbances and will reduce the resonant response at $\omega_{r}$ but it does introduce some sensitivity to fluctuations in ground tilt. However, with a suitable choice of damping factor, good isolation against tilts as well as horizontal disturbances can be achieved at angular frequencies above $\omega_{r}$ in most practical situations. Thus the use of a reference arm can provide a practical way of approaching the isolation obtained with a single pendulum of extremely long period.

There are, however, many experimental situations where this technique of correcting experimental data for seismic noise is not convenient, requiring, for example, large dynamic range in the measuring apparatus as well as high precision. In fact, there may be some cases where intrinsic freedom from disturbing accelerations is desirable. Adoption of a feedback isolation technique can be useful in such circumstances.

\section{An active system for isolating horizontal disturbances}

A system for achieving isolation against horizontal disturbances, which are the most important for current free mass gravitational wave detectors, has been under development at Glasgow for some time. In this section some of the basic ideas and preliminary experimental work are discussed. The basic concept is similar in principle to that of Erath et al, as reviewed by Melton (1957), for lengthening the period of a mass and spring seismometer by electronic means, although it was devised and developed independently. Our system operates by monitoring the relative horizontal displacement of the point of suspension of a test mass with respect to the mass itself and feeding back a suitably amplified and filtered form of this signal to a transducer which controls the position of the suspension point. To avoid coupling in tilts of the ground a reference arm is used and the relevant horizontal displacements are measured by monitoring the inclination of the suspension with respect to the reference arm (see figure 2). The equations of motion for this system in the

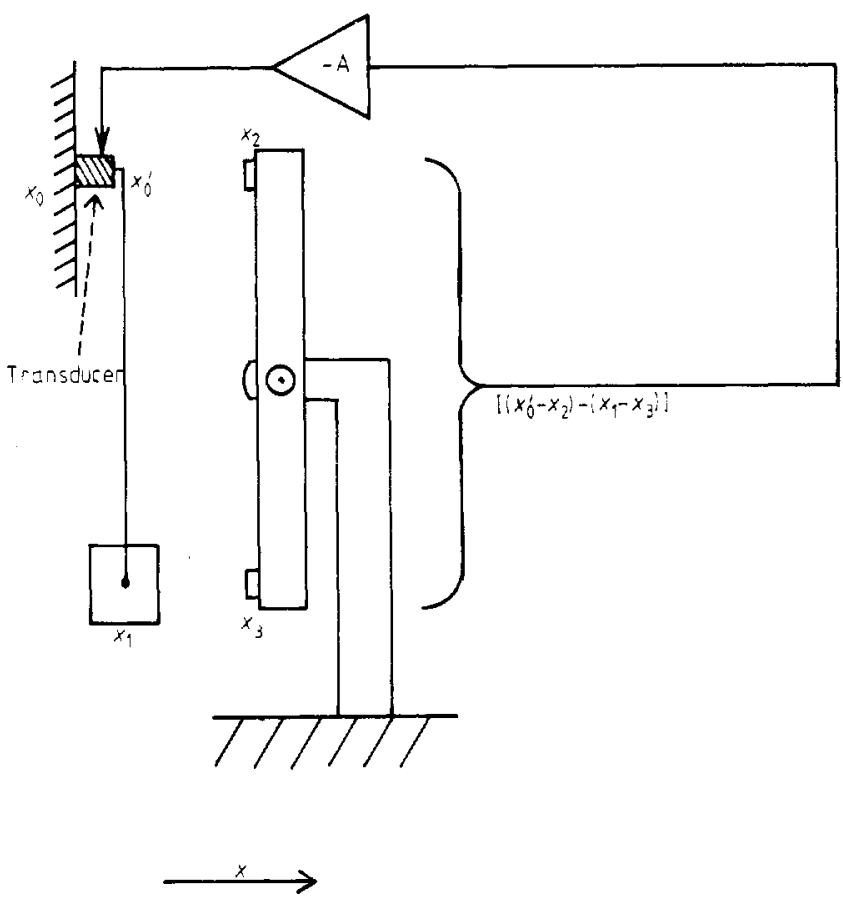

Figure 2. Schematic diagram of the anti-seismic feedback system discussed in the text. 
(a)

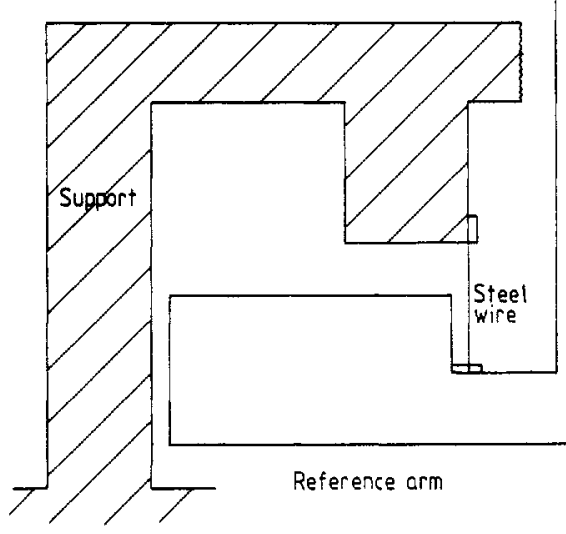

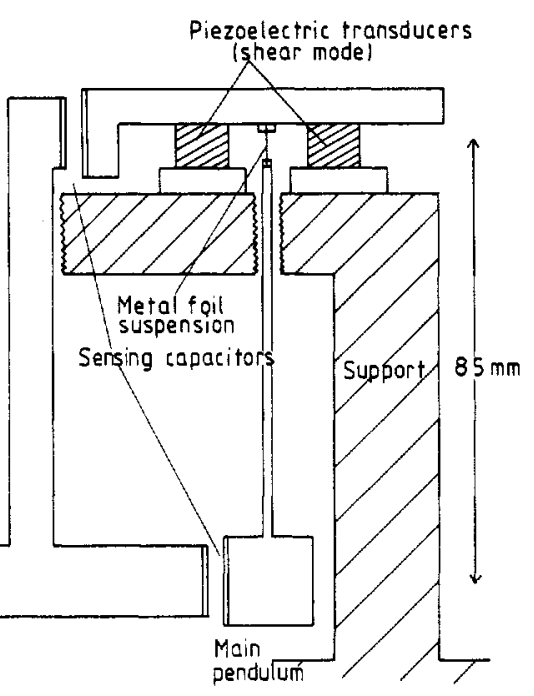
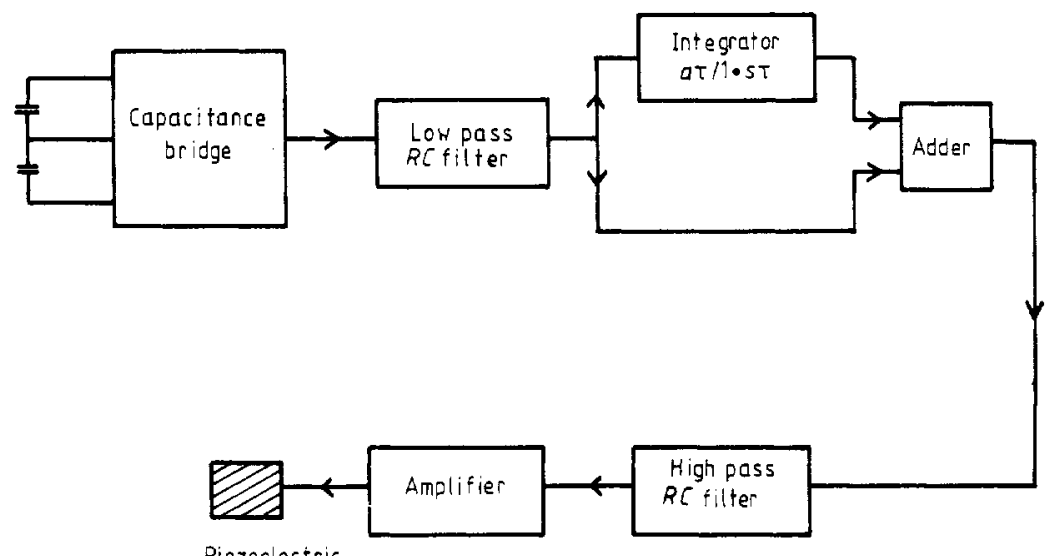

Piezolectric

transducers

(b)

Figure 3. (a), Simplified diagram of a test model of an active anti-seismic system incorporating a reference arm and capacitive sensing. The test mass $(0.47 \mathrm{~kg})$ was suspended by a rod and metal foil hinge to have a pendulum frequency of $1.7 \mathrm{~Hz}$. The reference arm was suspended by a wire attached to it at a point close to its centre of mass to give it a rocking frequency of $0.14 \mathrm{~Hz}$. (b), Block diagram of the feedback circuit for this system. The low-pass filter had a $3 \mathrm{~dB}$ point of $30 \mathrm{~Hz}$ and the high-pass filter a $3 \mathrm{~dB}$ point of $1.6 \times 10^{-3} \mathrm{~Hz}$. Parameters of the integrator are $\tau=10 \mathrm{~s}$ and $a=2 \mathrm{~s}^{-1}$ as discussed in the text.

absence of natural damping, and assuming a pendulum consisting of a point mass suspended by a wire of negligible mass, are as follows:

$$
\begin{gathered}
\ddot{x}_{1}=\omega_{0}^{2}\left(x_{0}^{\prime}-x_{1}\right) \\
x_{0}^{\prime}=x_{0}-A\left[\left(x_{0}^{\prime}-x_{2}\right)-\left(x_{1}-x_{3}\right)\right]
\end{gathered}
$$

where $x_{0}, x_{0}^{\prime}$ and $x_{1}$ represent the positions of the ground, point of suspension and test mass respectively; $x_{2}$ and $x_{3}$ represent the positions of the top and bottom reference points on the reference arm; and $\omega_{0}$ is the pendulum angular frequency. Since the movement $x_{2}-x_{3}$ of the reference arm can be made very small, by suitable choice of its natural period and damping, equation (2) can be approximated as

$$
x_{0}^{\prime}=x_{0}-A\left(x_{0}^{\prime}-x_{1}\right) .
$$

If the system is excited by a sinusoidal signal of angular frequency $\omega$, solution of these equations with $s=j \omega$ yields

$$
\begin{aligned}
& \frac{x_{0}^{\prime}}{x_{0}}=\frac{s^{2}+\omega_{0}^{2}}{s^{2}(1+A)+\omega_{0}^{2}} \rightarrow \frac{1}{A} \text { for } A \text { large } \\
& \frac{x_{1}}{x_{0}}=\frac{\omega_{0}^{2}}{s^{2}(1+A)+\omega_{0}^{2}} \rightarrow \frac{-\omega_{0}^{2}}{\omega^{2} A} \text { for } A \text { large. }
\end{aligned}
$$

It can be seen that the effect of the feedback is to cut down the response of the pendulum to ground motion. In fact as is indicated by equation (4) the active system behaves as a pendulum of new resonant angular frequency $\omega_{0}^{\prime}=\omega_{0} / \sqrt{1+A}$. It is desirable to introduce electronic damping into the system, and this may be achieved by using a feedback function of the form $A(1+(a / s))$ in place of $A$, although such a function can only be approximated in practice. It should be noted that damping is achieved by using the integral of the sensing signal and this may be understood by recalling that the sensing signal is proportional to the acceleration of the mass. It can be deduced 
from the above equations that to achieve critical damping, ' $a$ ' is given by $a \approx 2 \omega_{0}(A)^{-1 / 2}$ when $A \gg 1$. Adding damping in this manner does not affect the isolation achieved for large $A$ as expressed in equations (4) and (5).

We have here considered isolation against horizontal disturbances. For vertical disturbances the problem is different because it is much less important that a fixed reference direction be maintained. Here it is possible to suspend the test mass by a spring, sense changes in the extension of the spring, and use these to move the suspension point of the spring in a vertical direction in such a way as to maintain the spring at constant length (see Melton 1962). A vertical anti-seismic system of this type is presently being developed at JILA by Faller and Rinker (1979). It is clearly possible in principle to achieve seismic isolation down to very low frequencies in all three dimensions by combining this type of vertical spring isolation system with horizontal isolation in two orthogonal directions using a single reference arm suspended so that it can move freely in both directions, or using two separate reference arms.

A first test model of an active system, incorporating a reference arm and capacitive bridge sensing of the relevant displacements, is shown in figure $3(a)$. A block diagram of the feedback loop is given in figure $3(b)$. A number of points about this system should be noted. For initial testing of the basic ideas the reference arm in this case was suspended by a wire attached to a point close to its centre of mass to give a rocking frequency of $0.14 \mathrm{~Hz}$ and the test mass was suspended by a foil hinge connected to a rigid rod. This latter factor makes a negligible difference to the overall transfer function for the feedback system but it should be noted that a single foil hinge and rigid rod arrangement is not ideal for the suspension of the test masses in a gravitational wave detector as seismic isolation at high frequencies is poorer than with a wire suspension.

The transfer function from the driving point at the top of the main pendulum to the differential sensing point tends to zero at very low frequency. Hence any DC offset or very-low-frequency drift in the sensing system tends to drive the suspension point to one of its extremes. This could be dealt with by using a low-passfiltered version of the feedback signal to adjust the angle of the reference arm. An alternative approach, which was used in some tests, incorporated a high-pass $R C$ filter in the main feedback loop. The $3 \mathrm{~dB}$ point of this filter $\left(1.6 \times 10^{-3} \mathrm{~Hz}\right)$ was chosen to be much lower than the pendulum frequency of the test mass $(1.7 \mathrm{~Hz})$ to avoid feedback instability at high loop gain. The gain of the amplifier inside the analogue integrator providing damping was chosen to have a $3 \mathrm{~dB}$ point of $0.016 \mathrm{~Hz}$, well below the main pendulum frequency.

The system was operated with a loop gain $A$ of approximately 60 over a bandwidth of $30 \mathrm{~Hz}$ and the highfrequency gain coefficient of the integrator, $a$, was chosen to give critical damping ( $a \approx 2$ for $A=60$, for example). The value of gain-bandwidth product employed was limited by a resonance in the particular piezoelectric transducer assembly used for these tests, which was a pair of piezoelectric elements operating in shear mode. Figure 4 represents the differential sensing signal between the suspension point and the test mass of the pendulum, measured with respect to the reference arm, with the feedback loop open and closed. The signal is expressed in terms of displacement per square root of unit frequency interval.

The performance of this and other test systems was very encouraging. However when suspension wires are used for the test masses a possible problem in principle could be feedback instabilities arising from transverse 'violin mode' resonances in the suspending wires or propagation delays down the wires. The fact that the system senses the difference between bottom and top of the pendulum makes it easier to achieve stability, although a limit is set to the quality factor, $Q$, which can be

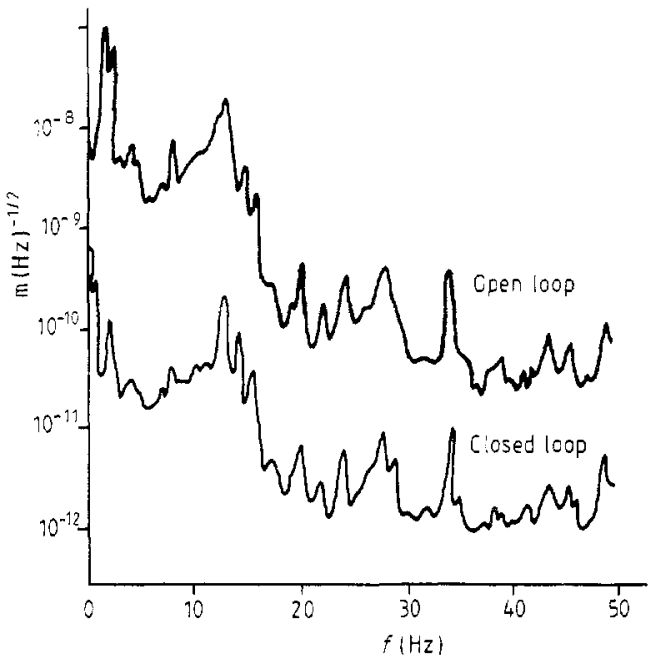

Figure 4. Spectral density of the differential displacement signal from the apparatus of figure 3 . The signal is shown for the feedback loop open and closed.

allowed for the wire resonances. This is dependent on the other parameters of the system and reasonably high values of $Q$ are permissible. For example, for a mass suspended by a $0.3 \mathrm{~m}$ long hardened steel wire, under a stress of $1.5 \times 10^{9} \mathrm{Nm}^{-2}$ (giving a first wire resonance at $730 \mathrm{~Hz}$ ), in a feedback system with a loop gain of 100 rolled off by a simple $R C$ filter of $3 \mathrm{~dB}$ point at $1 \mathrm{kHz}$, the $Q$ for the first wire resonance can be allowed to exceed $5 \times 10^{5}$ (Robertson 1981). It may be remarked that high $Q$ is usually desirable for gravity wave detectors to minimise thermal noise, but in other applications wire resonances might be removed by suitable damping.

\section{Application to a gravitational wave detector}

In the application of this active anti-seismic system to our gravitational wave detector which uses $10 \mathrm{~kg}$ test masses suspended by wires, a cross spring hinge suspension of the reference arm is adopted to give it a rocking period in excess of $30 \mathrm{~s}$. There are advantages in using optical sensing instead of capacity transducers as there is less danger of significant electrostatic forces in an optical system and the arrangement can also be made less sensitive to precise positioning of the test mass with respect to the reference arm - an important practical advantage. One way of achieving the required differential displacement sensing is to use a simple Michelson interferometer, as indicated schematically in figure 5 . With a lowpower helium-neon laser $(\sim 1 \mathrm{~mW})$ the photon noise limited displacement sensitivity of such a system is approximately $2 \times 10^{-15} \mathrm{~m}(\mathrm{~Hz})^{-1 / 2}$. However, if a simple fringe sensing is used, a linear signal is obtained only if the differential motion to be measured is small compared with the wavelength of the light used. A more elaborate arrangement which counts passing fringes, taking account of their direction of movement, is desirable if larger movements are to be dealt with. Work on systems of this kind is currently being carried out.

\section{Conclusion}

We have described here seismic isolation techniques which seem capable of providing effective isolation at relatively low frequencies. For high-frequency isolation the simpler and well known passive isolation systems appear satisfactory. A suitable 


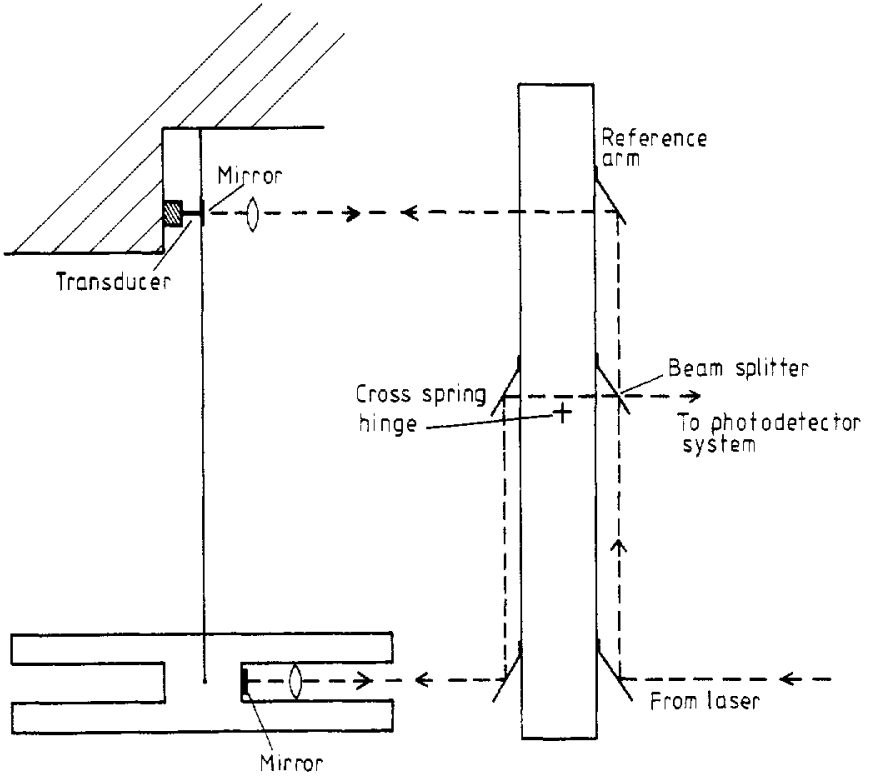

Figure 5. Schematic diagram of a possible suspended mass and reference arm system with optical interferometric sensing.

combination of one or more stages of the type of active isolation system discussed here together with several stages of suitable passive isolation would seem capable of providing the large degree of wideband seismic isolation required for planned gravitational radiation detectors. Applications in geophysics and other fields of sensitive physical measurements also seem likely.

\section{Acknowledgments}

We acknowledge the financial support of the University of Glasgow and the Science and Engineering Research Council. We wish to thank R V Pound, Harvard University, for helpful private communications on some aspects of the system and D Murray Smith, Glasgow University, for useful advice on control systems.

\section{References}

Drever R W P, Ford G M, Hough J, Kerr I, Munley A J, Pugh J R, Robertson N A and Ward H 1980 A gravity wave detector using optical cavity sensing

Proc. 9th Int. Conf. on General Relativity and Gravitation, GR9, Jena in press

Drever R W P, Hough J, Munley A J, Lee S A, Spero R, Whitcomb S E, Ward H, Ford G M, Hereld M, Robertson N A, Kerr I, Pugh J R, Newton G P, Meers B, Brooks E D III and Gursel Y 1981 Optical cavity laser interferometers for gravitational wave detection Laser Spectroscopy $V$ ed. A McKellar et al (Berlin: Springer) p 33

Faller J E and Rinker R L 1979 Super-Spring Dimensions September $\mathrm{p} 25$

Lorenzini D A 1972 Active control of a pneumatic isolation system

Proc. AIAA Guidance and Control Conference, Stanford, California, Paper No 72-843

Melton B S 1957 Contributions to seismology and geomagnetism $A d v$. Electron. and Electron Phys. (New York: Academic) IX 297
Melton B S and Johnson D P 1962 Inertial seismograph design - limitations in principle and practice Proc. IRE 502328

Robertson N A 1981 Experiments relating to the detection of gravitational radiation and to the suppression of seismic noise in sensitive measurements PhD Thesis University of Glasgow, Scotland

Thorne K S 1980 Gravitational-wave research: current status and future prospects

Rev. Mod. Phys. 52285

Weber J 1969 Evidence for discovery of gravitational radiation Phys. Rev. Lett. 221320

Weiss R 1972 Electromagnetically coupled broadband gravitational antenna Quarterly Progress Report, Research Lab. of Electronics MIT 10554 